\title{
Psychodynamische Therapie der Depression
}

\author{
Dorothea Huber, Günther Klug
}

\section{Übersicht}

Einleitung

Psychodynamisches Ätiologiekonzept

Psychodynamische Psychotherapie

Therapie der akuten Depression

\section{Einleitung}

\section{Epidemiologie}

Patienten mit depressiven Störungen stellen einen sehr großen Teil der Behandelten sowohl in ambulanter als auch in stationärer Psychotherapie. Das große persönliche Leid der Patienten, aber auch die gesellschaftlichen Folgekosten, unterstreichen die Bedeutung der Behandlung von Depressionen. Nach Schätzung der WHO wird die Depression 2020 weltweit die zweithäufigste Erkrankung sein. Die Wahrscheinlichkeit, im Laufe des Lebens eine Depression zu erleiden, liegt bei bis zu 12\% für Männer und bis zu 26\% für Frauen. Häufig wird in den letzten Jahren deshalb von der „Volkskrankheit Depression“ gesprochen.

Ergebnisse epidemiologischer Forschung zeigen, dass die Depression meist eine wiederkehrende Störung ist, die für eine beträchtliche Anzahl von Patienten (10-30\%) chronisch wird. Zudem ist die Überlappung mit anderen Störungen, insbesondere mit Angststörungen, somatoformen Störungen und Persönlichkeitsstörungen sehr hoch; die Leitlinie Depressionsbehandlung spricht von 75-90\% Komorbidität [1]. Screeninguntersuchungen in Allgemeinarztpraxen und Kliniken verweisen auf erhebliche Quoten von depressiven Störungen, die hinter anderen Beschwerden verborgen sind und oft unerkannt bleiben [2].
Die Depression nimmt für 10-30\% der Patienten einen chronischen Verlauf mit häufig hoher Komorbidität.

Auch sind die Rückfallquoten für jede Form von Kurzpsychotherapie erschreckend hoch [3], was zu einem neuen Interesse an psychodynamischen Langzeitbehandlungen geführt hat. In der psychodynamischen Psychotherapie stellt die Depression die größte Krankheitsgruppe dar. Rudolf beschreibt, dass bei $80 \%$ der Patienten in ambulanter psychodynamischer Psychotherapie depressive Symptome vorliegen [4].

\section{Diagnose und Differenzialdiagnose}

Typische Beschwerden einer Depression sind depressive Stimmung, Antriebsminderung, Interessenverlust, Freudlosigkeit, Dysphorie, Energielosigkeit, Unruhe, Konzentrationsstörungen, vermindertes Selbstwertgefühl, Selbstzweifel, Schuldgefühle, Hilflosigkeit, Hoffnungslosigkeit und Sinnlosigkeit sowie Suizidideen, Appetitstörung, Gewichtsverlust, Libidoverlust, Schlafstörung und körperliche Missempfindungen. Wenngleich viele der genannten Gefühlszustände und Beschwerden allen Menschen bekannt sind, liegt bei Überschreiten einer bestimmten Intensität bzw. Dauer ein depressives Syndrom vor, das dann die Kriterien für die Diagnose einer Depression erfüllt. 
Mit psychodynamischer Psychotherapie werden hauptsächlich Patienten mit den folgenden Diagnosen behandelt:

- depressive Episode (F32)

- rezidivierende depressive Störung (F33)

- Dysthymia (F34.1)

- rezidivierende kurze depressive Störung (F38.1)

- Anpassungsstörung, längere depressive Reaktion (F43.21)

Es handelt sich also um Patienten aus den diagnostischen Kategorien F3: affektive Störungen und F4: neurotische, Belastungs- und somatoforme Störungen im ICD-10.

Bei der depressiven Episode und der rezidivierenden depressiven Störung wird jeweils zwischen leichter, mittelgradiger und schwerer depressiver Episode unterschieden. Die Dysthymia ist durch leichtere Symptome als die depressive Episode gekennzeichnet, aber auch durch langdauernde depressive Verstimmung (mindestens 2 Jahre), d.h. durch einen chronischen Verlauf. Wenn die Kriterien für eine depressive Episode erfüllt sind, muss nach ICD-10 diese Diagnose gestellt werden, auch wenn gleichzeitig eine Dysthymia vorliegt; im DSM-IV besteht die Möglichkeit, eine „Double Depression“ zu diagnostizieren - dies hat insofern Bedeutung, als diese Form der Depression als die am schwersten behandelbare gilt [3].

Die Depressiven, die in die psychotherapeutischen Ambulanzen oder Praxen kommen, haben in aller Regel eine jahrelange Vorgeschichte von depressivem Leiden und verschiedenen Behandlungsversuchen hinter sich. Oft ist ihnen durch antidepressive Medikation oder Kurzzeitpsychotherapie vorübergehend geholfen worden, doch die Beschwerden sind wiedergekehrt.

Das typische Klientel der analytischen Psychotherapie leidet an chronifizierten Krankheitsbildern.

Nach Leuzinger-Bohleber et al. sind es die besonders schweren, chronifizierten und behandlungsresistenten („difficult-to-treat“) Depressionen, an denen sich die analytische Psychotherapie beweisen sollte [5].

\section{Die Nationale Versorgungsleitlinie}

Da die Behandlung der Depression ein sehr wichtiges Thema geworden ist, liegt seit 2009 nunmehr eine S3Leitlinie bzw. die Nationale Versorgungsleitlinie (NVL) zur Behandlung der unipolaren Depression Erwachse-

\section{Tipp für die Praxis}

Es hat sich in der psychodynamischen Diagnostik durchgesetzt, neben einer Symptom- auch eine Strukturdiagnose (also eine Einschätzung des Niveaus der Persönlichkeitsorganisation) zu stellen. In einer bundesweiten Standardisierung von psychodynamischen Diagnosen mit dem Operationalisierten Psychodynamischen Diagnosesystem OPD-2 wurde auch die Strukturdiagnose als Achse-IV-Struktur miteinbezogen [6].

ner vor [1]. Diese NVL wurde in einem mehrjährigen Prozess von über 30 ärztlichen und psychologischen Fachgesellschaften und Berufsverbänden erarbeitet.

Diese Leitlinien übernehmen zunehmend die Funktion eines zu erfüllenden Mindestqualitätsstandards bei der Behandlung depressiver Patienten.

Der Geltungsbereich der NVL ist auf die unipolare depressive Störung Erwachsener, d.h. depressive Episoden, rezidivierende depressive Störungen, anhaltende affektive Störungen (hier nur: Dysthymia) und sonstige affektive Störungen (hier nur: rezidivierende kurze depressive Störung) beschränkt. Die Aussagen der Leitlinien beziehen sich auf Ätiologiemodelle, diagnostische Vorgehensweisen, allgemeine Behandlungsgrundlagen sowie Pharmako- und Psychotherapie einschließlich Erhaltungstherapie und Rezidivprophylaxe. Darüber hinaus wird das Management bei Suizidgefahr beschrieben.

\section{Rolle der Psychotherapie}

\section{Fazit der Leitlinie:}

Es wird explizit darauf hingewiesen, dass Psychotherapien einen deutlich höheren „Carry-over“-Effekt haben, also eine längerfristige Wirkung noch nach beendeter Behandlung. Auch belegen Studien, dass die beste Rückfallprophylaxe, auch nach einer erfolgreich abgeschlossenen Pharmakotherapie, eine anschließende Psychotherapie ist.

Zusammengefasst lauten die Empfehlungen der Leitlinie:

Leichte depressive Episoden. Es wird davon ausgegangen, dass bei leichten depressiven Episoden eine Pharmakotherapie nicht Mittel der ersten Wahl ist, es sei denn, es besteht ein entsprechender Wunsch des 
Patienten, ausgesprochen positive Vorerfahrungen oder eine Restsymptomatik nach anderen Interventionen bzw. aus der Anamnese bekannte mittelgradige oder schwere Depressionen. Ansonsten gilt Psychotherapie als Mittel der ersten Wahl.

Mittelgradige Depressionen. Bei mittelgradigen Depressionen wird im akuten Stadium von einer gleichen Wertigkeit von antidepressiver Medikation und Psychotherapie ausgegangen. Die Empfehlungen sehen beide Behandlungsmöglichkeiten vor.

Schwere Depressionen. Bei schweren Depressionen wird dezidiert empfohlen, sowohl Psychopharmakotherapie als auch Psychotherapie anzubieten. Alle Antidepressiva können als äquivalent wirksam angesehen werden. Im Bereich der Psychotherapie werden alle in Deutschland in der Richtlinienpsychotherapie verankerten Therapieverfahren empfohlen.

\section{Tipp für die Praxis}

Es wird empfohlen, sich vor der Behandlung von depressiven Patienten mit der S3-Leitlinie Depression vertraut zu machen.

\section{Psychodynamisches Ätiologie- konzept}

Der depressive Grundkonflikt und seine Verarbeitung als psychische Disposition einer späteren depressiven Erkrankung ist durch folgende psychodynamische Konstellation charakterisiert: Die zentrale Beziehungserfahrung des Verlassenwerdens und/oder des Verlustes mit dem charakteristischen Gemisch aus Objektbedürftigkeit und Objektenttäuschung ist aufgrund struktureller Unreife des Depressiven emotional unerträglich. Starke Bemühungen, sich dem Objekt anzubieten, um es zurückzugewinnen, sind die Folge. Alles was die Beziehung zum Objekt gefährden könnte, wird vermieden. Stattdessen wird versucht, ein ideales Bild des Objekts aufrechtzuerhalten (Idealisierung) und die Wahrnehmung der emotional heftigen Objektenttäuschung wird vermieden. Diese Vorgänge sind verbunden mit einer extremen Anstrengung und bedeuten psychophysiologisch permanenten Stress durch die ständigen Bindungsbemühungen, Verlustängste, Anpassungsbereitschaft und andrängende Enttäuschungswut.
Der depressive Grundkonflikt beinhaltet Verlust des Objekts oder die Enttäuschung am Objekt. Das typische Bewältigungsmuster (Abwehr) ist die Wendung der Aggression gegen das Selbst.

Rudolf stellt beim depressiven Grundkonflikt die folgenden 3 Aspekte in den Mittelpunkt [4]:

1. die Internalisierung von konflikthaften Beziehungsmustern (Beziehungskonflikte, Bindungsprobleme),

2. die Entwicklung struktureller Vulnerabilität und

3. die Ausbildung spezifischer dysfunktionaler Bewältigungsmuster.

Am Beispiel der depressiven Störungen verdeutlicht er, wie Konflikt und Struktur beide Aspekte einer früheren Bindungserfahrung darstellen. Auf der Grundlage der frühen Verluste bzw. Defiziterfahrung besteht ein ausgeprägtes Bedürfnis nach nur guter Beziehung. Bei einerseits Sehnsucht nach dem idealisierten Objekt und andererseits der Unfähigkeit, Aggression direkt gegen das enttäuschende Objekt zu richten, wird, als Hauptabwehrmechanismus der Depressiven, die Aggression gegen das eigene Selbst gewendet. Rudolf betont, dass bei der Entwicklung der Depression sowohl der konflikthafte Beziehungsmodus als auch die strukturelle Vulnerabilität eine Rolle spielen. Die strukturelle Vulnerabilität betrifft Schwierigkeiten der SelbstObjekt-Differenzierung und ganzheitlichen Objektwahrnehmung sowie der Loslösung von Objekten, der Selbstwertregulierung, der Affektsteuerung und Affektdifferenzierung.

\section{Psychodynamische Psycho- therapie}

Da der Begriff der psychodynamischen Psychotherapie unterschiedlich gebraucht wird, wollen wir im Folgenden darstellen, welche Definition wir zugrunde legen. Es setzt sich zunehmend durch, psychodynamische Psychotherapie als Überbegriff für die in der Richtlinienpsychotherapie definierten Therapieverfahren zu verstehen:

- analytische Psychotherapie und

- tiefenpsychologisch fundierte Psychotherapie.

Psychodynamische Psychotherapie ist der Oberbegriff für analytische Psychotherapie und tiefenpsychologisch fundierte Psychotherapie.

Analytische Psychotherapie. Die analytische Psychotherapie, mit in der Regel 3 Wochenstunden und meist im Liegen durchgeführt, gewinnt ihr Material v.a. 
durch die freien Assoziationen des Patienten, denen der Therapeut seine „gleichschwebende“ Aufmerksamkeit gegenüberstellt. Die aktuellen Konflikte und strukturellen Defizite werden vor dem Hintergrund der pathogenetisch bedeutsamen Beziehungen zu den primären Bezugspersonen erhellt. Die interpersonellen Probleme, die den Symptomen und Beziehungsstörungen zugrunde liegen, werden an den intrapsychischen, unbewussten Selbst- und Objektrepräsentanzen bearbeitet, wobei die Wiederholung dieser Beziehungsmuster in der Übertragung auf den Therapeuten die zentrale therapeutische Strategie ist, um sie im Hier und Jetzt der therapeutischen Beziehung effektiv bearbeiten zu können.

Gemäß den Psychotherapierichtlinien übernehmen die Krankenkassen bei nachgewiesener Indikation und ausreichend guter Prognose die Kosten der analytischen Psychotherapie derzeit bis max. 240 Behandlungsstunden.

Tiefenpsychologisch fundierte Psychotherapie. Die tiefenpsychologisch fundierte Psychotherapie bezeichnet eine Behandlungsform, die sich an der psychoanalytischen Krankheitstheorie und Behandlungstechnik orientiert. Sie unterscheidet sich aber durch ihre inhaltliche Schwerpunktsetzung sowie durch die niedrigere Anzahl und Frequenz der Therapiesitzungen als externes Kriterium von der psychoanalytischen Behandlungsmethode. Auch sie gewinnt ihr Material durch die freien Assoziationen des Patienten, die aber durch Frequenz und Dauer der Therapie als auch durch die Fokussierung als technisches Kriterium eingeschränkt sind. Zentrales Merkmal der Theorie der Technik ist die Fokussierung auf innere, meist unbewusste Konflikte, die als auslösend bzw. bedingend für eine aktuelle psychische Symptomatik gesehen werden (Aktualkonflikte) und damit auf eine Begrenzung regressiver Prozesse.

Die Technik der Fokussierung auf innere Konflikte ist ein zentrales Merkmal der tiefenpsychologisch fundierten Psychotherapie.

Thematisch konzentriert sich die tiefenpsychologisch fundierte Psychotherapie besonders auf die pathogene Beziehungsgestaltung der Patienten zu wichtigen Bezugspersonen meistens der frühen Kindheit. Die interpersonellen Probleme, die den Symptomen und Beziehungsstörungen zugrunde liegen, werden im interpersonellen Raum bearbeitet, ohne dass eine vertiefte Analyse der unbewussten Selbst- und Objektrepräsentanzen stattfindet. Wie in der analytischen Psychothe- rapie wird davon ausgegangen, dass sich die pathogenen Konflikte und Probleme in der therapeutischen Beziehung reproduzieren; in der Regel dient ihre Kenntnis aber nur der Orientierung des Therapeuten und sie werden nicht zusammen mit dem Patienten analysiert. Eine direkte Arbeit an und mit der Übertragung im Rahmen der therapeutischen Beziehung erfolgt, wenn sie nötig ist, z. B. bei der Widerstandsanalyse, sie ist aber nicht die bevorzugte Behandlungsstrategie.

Der Schwerpunkt der Behandlung liegt auf der Auseinandersetzung mit dem gegenwärtigen Leben: den auslösenden Konflikten und den aktuellen beruflichen, sozialen, familiären und Beziehungsproblemen.

Die tiefenpsychologisch fundierte Psychotherapie wird in der Regel mit einer Wochenstunde und im Sitzen durchgeführt; die Krankenkassen übernehmen die Kosten derzeit bis zu 80 Behandlungsstunden.

Im Folgenden werden die beiden Verfahren der psychodynamischen Psychotherapie näher erläutert.

\section{Therapie der akuten Depression}

\section{Allgemeine Prinzipien}

Der Umgang mit der akuten Depression, der häufig krisenhaft und dramatisch abläuft, wird von vielen psychotherapeutischen und psychiatrischen Schulen ähnlich gesehen und wird im Folgenden in 13 Punkten überblicksartig dargestellt. Die Ausführungen sind angelehnt an Schauenburg [7].

- Das unaufdringliche Zuhören und die Bereitstellung von Zeit und Raum stellt eine erste Beruhigung des depressiven Patienten dar.

- Da depressive Patienten im bewussten Erleben davon ausgehen, dass ihnen Aufmerksamkeit nicht zusteht, empfiehlt es sich, sie darin zu bestärken, dass sie sich Hilfe gesucht haben.

- Eine genaue Erhebung des Ausmaßes der depressiven Symptomatik ist nicht nur zu diagnostischen, sondern auch zu therapeutischen Zwecken gut, da sie Verständnis signalisiert.

- Die diagnostische Klärung dient nicht nur der eigenen Orientierung, sondern ist oft ein direkter Weg, den Betroffenen Akzeptanz und Verständnis zu vermitteln. 
- Das Suizidrisiko muss als unabdingbarer Bestandteil der initialen Diagnostik auf jeden Fall aktiv erfragt und ggf. müssen Maßnahmen ergriffen werden.

- Selbst wenn nach klinischer Einschätzung eine langfristige Therapie angezeigt ist, kann es anfangs sinnvoll sein, zunächst nur einen kürzeren Behandlungsabschnitt (ca. 20 Stunden) zu vereinbaren, um evtl. Abhängigkeitsängste nicht zu sehr zu stimulieren.

- Es empfiehlt sich, eine evtl. nötige Begleitmedikation und deren Nebenwirkungen auch schon am Anfang zu besprechen, damit der Patient nicht unter der Therapie den Eindruck erhält, der Therapeut wisse mit psychotherapeutischen Methoden nicht mehr weiter.

- Es ist empfehlenswert, dem Patienten möglichst viel Information über Symptomatik und Erkrankung zu vermitteln.

- Es sollte möglichst am Anfang auch nach den Therapieerwartungen des Patienten gefragt werden.

- Klare Absprachen am Beginn einer Behandlung sind unbedingt erforderlich.

- Es kann sehr hilfreich sein, mit dem Patienten gemeinsam sog. antidepressive Strategien zu erarbeiten wie Sport oder Aufnahme sozialer Kontakte.

- Der Therapeut kann durchaus seine eigene Wahrnehmung im Sinne eines Hilfs-Ichs zur Verfügung stellen.

- Der Therapeut sollte einen Behandlungsfokus erstellen, beispielsweise ein problematisches Beziehungsmuster, wie altruistische Abtretung oder strukturelle Einschränkungen, wie gestörte Affektregulation. Den ausgewählten Fokus kann man sowohl als innere Richtschnur des Therapeuten im Verlaufe der Psychotherapie benennen als auch als eine Hypothese dem Patienten mitteilen - beides kann sehr entlastend wirken.

Insgesamt hat der Therapeut im Umgang mit akut Depressiven keine leichte Aufgabe, denn er muss einerseits eine betreuende und schützende Funktion ausüben, andererseits die erlebte Hilflosigkeit, den Pessimismus und die Distanz zum inneren Erleben aushalten und damit den Patienten stützen.

\section{Tipp für die Praxis}

Zur Stabilisierung des Therapieerfolgs sowie zur Senkung des Rückfallrisikos soll im Anschluss an eine Akutbehandlung eine angemessene psychotherapeutische Nachbehandlung (Erhaltungstherapie) angeboten werden. Dies empfiehlt auch die Nationale Versorgungsleitlinie Unipolare Depression.

\section{Analytische Psychotherapie}

Die analytische Psychotherapie Depressiver ist auf mehrere Jahre angelegt und findet in der Regel mit 3 Stunden pro Woche im Liegen auf der Couch statt. Bei Depressiven mit gering oder mäßig integriertem Strukturniveau nach OPD-2 empfiehlt es sich, mit 2 Wochenstunden im Sitzen zu arbeiten. Durch die Dauer und Intensität der analytischen Arbeit können Effekte erzielt werden, die weit über eine symptomatische Veränderung hinausgehen und zu einer strukturellen Veränderung, d.h. zu einer Veränderung der Konflikte und strukturellen Störungen führen, die der Symptomatik zugrunde liegen. Die strukturelle Veränderung wird als therapiespezifisches Ergebnis von psychoanalytischen, und in einem geringeren Umfang von tiefenpsychologisch fundierten Therapien angesehen. Die pathogenen Beziehungsmuster der Depressiven werden in der Übertragung auf den Therapeuten dargestellt und können dadurch im Hier und Jetzt verstanden, gedeutet und durchgearbeitet werden.

Als Faustregel gilt: Je chronischer die Erkrankung, je konfliktreicher das Leben und je differenzierter die Innenwelt des Patienten, desto klarer ist die Indikation für eine analytische Psychotherapie zu stellen.

Bei Depressiven, deren Symptomatik auf tiefgehenden Konflikten und auf Persönlichkeitsstörungen basiert, wird ausreichende Veränderung folgendermaßen ermöglicht [8]:

- durch das Setting geförderte regressive Prozesse

- Inszenierung der pathologischen Beziehungsmuster in der Übertragung

- Umwandlung der Charakterabwehr in aktuelle und bearbeitbare Widerstände

- Aktualisierung frühkindlicher, präverbaler Traumata und damit zusammenhängender Handlungsmodelle

- Veränderung durch die „neue Erfahrung“ mit dem Analytiker

- Behandlungstechnik, bei der auf der Basis des empathischen Zuhörens die Analyse der aktuellen Beziehung zwischen Analytiker und Patient, das Wissen um die Übertragung, die Verwendung der Gegenübertragung und die deutende Tätigkeit (inkl. der Übertragungsdeutung) im Vordergrund stehen.

Übertragung. Der zentrale unbewusste Konflikt wird dabei allmählich aktiviert, bestimmt eine Zeit lang die Übertragung - für beide Beteiligte oft schwer erträglich - und wird dadurch der Deutungsarbeit zugänglich. Das wesentliche Material, anhand dessen sich die Übertragung konkretisiert, können die Beziehungen zum 


\section{Analytische Psychotherapie}

\section{Wichtige Ziele}

- negative Beziehungserwartungen in der Übertragungsbeziehung aktualisieren (Analyse der „negativen Übertragung“)

- destruktive Fantasien erlebbar machen und dadurch die in ihnen liegenden potenziell positiven Kräfte freilegen

- Vermeidung bzw. altruistische Abtretung eigener Bedürfnisse in wahrnehmbare Triebwünsche und Bedürfnisse verändern
- aus der chronischen Enttäuschung die Enttäuschungswut befreien

- die Hoffnungslosigkeit in eine offen zugelassene Hilfesuche transformieren

- destruktive Schuldgefühle in eine zunehmende Fähigkeit zur aktiven Beziehungsgestaltung umwandeln
Partner, zur Familie und am Arbeitsplatz, das Selbsterleben, Hindernisse und Hemmungen, verdrängte Wünsche in Träumen und Tagträumen usw. sein.

Veränderungsrelevanter Faktor. Als veränderungsrelevanter Faktor in der analytischen Behandlung wirkt weniger die kognitive Einsicht in die unbewusst motivierte und automatisierte Verhaltenspathologie und ihre lebensgeschichtliche Entwicklung, als das Erleben und die affektiv geladene Erinnerung der schmerzlichen Ursprungssituationen.

Durcharbeiten. Schließlich folgt das Durcharbeiten, d.h. das immer wieder neue „Durchkauen“ der zentralen Konflikte in ihren verschiedensten Erscheinungsformen, bis eine stabile Veränderung des Erlebens und Verhaltens des Depressiven erreicht ist. In aller Regel kommt es dann zu einer anhaltenden Auflösung der Symptomatik.

Insgesamt sollen durch die Arbeit in der analytischen Psychotherapie an und in der Übertragung mit depressiven Patienten deren typische Konfliktund Beziehungsmuster erkannt und „verflüssigt“ werden.

\section{Cave}

Eine besondere Gefahr in der analytischen Psychotherapie Depressiver ist das zu frühe Ansprechen der Aggressivität, ohne dass der Abwehrmechanismus der Wendung der Aggression gegen das eigene Selbst ausreichend bearbeitet wurde. Dann nämlich kann es zu Schuldgefühlen und zur Suizidalität kommen und im weiteren Verlauf auch durch die zunehmende negative Übertragung zum Therapieabbruch.

\section{Tiefenpsychologisch fundierte Psycho- therapie}

Zeitbegrenzung. Die tiefenpsychologisch fundierte Psychotherapie von depressiven Patienten findet mit einer Wochenstunde im Sitzen statt und basiert prinzipiell auf der gleichen Theorie der Technik wie die analytische Psychotherapie. Die Zeitbegrenzung bewirkt aber auf der Seite des Patienten, dass die freien Assoziationen in der Regel weder so weit vernetzt noch so tiefreichend wie in der analytischen Psychotherapie sein werden, auf der Seite des Therapeuten, dass seine gleichschwebende Aufmerksamkeit durch eine aktivere, fokussierte Haltung eingeschränkt ist. Dies hat Auswirkungen v.a. auf die Bearbeitung der gegen das eigene Selbst gewendeten Aggressivität, die von vielen psychoanalytischen Schulen als pathognomonisch angesehen wird.

Die Zeitbegrenzung beeinflusst die Möglichkeiten der Übertragung durch den Patienten und deren Deutung durch den Therapeuten.

Durch die zeitliche Limitierung wird die Aggressivität häufig nicht an und in der Übertragung auf den Therapeuten bearbeitet werden können, und damit entfällt die Übertragungsdeutung als Intervention, die seit langem als veränderungsrelevant angesehen wird [9], was für die strukturell gestörten Patienten empirisch belegt werden konnte [10]. Stattdessen wird ein adäquaterer Umgang mit der Aggressivität mehr durch die primäre Identifizierung mit dem Therapeuten erworben werden und durch die korrigierenden emotionalen Erfahrungen mit ihm; ein Weg, der auch zu stabilen, strukturellen Veränderungen führen kann, wie die Ergebnisse der Menningerstudie zeigen [11]. 
Adäquater Umgang mit der Aggressivität wird durch eine primäre Identifizierung mit dem Therapeuten und durch korrigierende emotionale Erfahrungen erworben.

Ein alternativer Weg ist die Arbeit an der Aggressivität in der Außenübertragung, bei der allerdings das zwingende Evidenzgefühl des Hier und Jetzt fehlen kann, das durch ein Dort und Damals nicht zu ersetzen ist. Grundlegend bleibt das therapeutische Dilemma, dass die Zeitspanne, um eine positive Übertragung für ein stabiles Arbeitsbündnis sich entwickeln zu lassen, benötigt wird und dass sie durch keine Manipulation des Therapeuten abzukürzen ist, und dass andererseits dann die Zeit für die Bearbeitung der Aggressivität nicht ausreicht. Darin ist eine Ursache für die empirisch belegte geringere Effektivität der tiefenpsychologisch fundierten gegenüber der analytischen Psychotherapie zu sehen [12].

Häufig wird in der tiefenpsychologischen Psychotherapie die Aggressivität in der Außenübertragung bearbeitet.

Arbeit mit Emotionen. Ein weiteres Problem der tiefenpsychologisch fundierten Psychotherapie bei depressiven Patienten kann darin bestehen, dass der Patient zwar intellektuelle Einsichten gewinnt, deren Umsetzung in Veränderungen des Selbsterlebens und Verhaltens im Bereich der für Depressive so wichtigen negativen Emotionen aber nur unzureichend gelingt, weil die ganze Skala der aggressiven Gefühle nicht in das Selbst integriert werden konnte. Die dann erreichten Veränderungen sind nicht stabil, da Emotionalität und Erleben zu kurz gekommen sind, und sie halten der wirklichen Prüfung auf Stabilität durch die Konfrontation mit den zentralen Konflikten nicht stand.

Von besonderer therapeutischer Bedeutung in der tiefenpsychologisch fundierten Psychotherapie von depressiven Patienten ist der Umgang mit den positiven Selbstanteilen, den Ressourcen und den Bewältigungsstrategien [4]. Aus den unterschiedlichsten Motiven muss der Depressive sie verbergen und damit unbrauchbar machen (z.B. durch Entwertung). Hier ist es wichtig, vor dem stets mitzudenkenden, unbewussten Hintergrund, diesen Bereich therapeutisch nicht als Abwehr infrage zu stellen, sondern als Leistung des Patienten anzuerkennen. Die Verarbeitungs-/Bewältigungsmuster dienen dazu, sich selbst und die Beziehung zum anderen so zu modifizieren, dass die depressiven Affektspannungen erträglich werden.
Wichtig ist der Umgang mit den positiven Selbstanteilen, den Ressourcen und Bewältigungsstrategien.

\section{Tipp für die Praxis}

Es ist wichtig sich klar zu machen, dass nicht das depressive Symptom behandelt wird, sondern die Psychodynamik des interpersonellen Verhaltens, d.h. die dysfunktionalen Beziehungen bzw. unsicheren Bindungen, die den Symptomen zugrunde liegen.

Haltung des Therapeuten. Rudolf beschreibt die therapeutische Haltung, die als Hintergrund für die Interventionen des tiefenpsychologisch fundiert arbeitenden Therapeuten dienen soll [4]. Er sollte:

- als ausreichend gutes Objekt, d.h. als zuverlässiges Gegenüber zur Verfügung stehen;

- als Hilfs-Ich dienen, welches bei der Affektwahrnehmung und Affektdifferenzierung unterstützt, zur Affekttoleranz anleitet und im Ertragen der nicht erfüllten Bedürftigkeit begleitet (Frustrationstoleranz);

- auch als Hilfs-Über-Ich dienen, welches sich gegen die Selbstentwertung stellt und bei der adäquaten Selbstbewertung unterstützt;

- sich als antwortendes Gegenüber verhalten, das sich getrennt vom Patienten verhält und erlebt (SelbstObjekt-Differenzierung);

- sich als interessiert an den Mitteilungen des Patienten zeigen, an der Weiterentwicklung des Patienten und an der Unterbindung von Selbstschädigungstendenzen;

- interessiert an einer gemeinsamen therapeutischen Zielsetzung sein.

\section{Fazit für die Praxis}

Insgesamt ist das therapeutische Vorgehen in der tiefenpsychologisch orientierten Psychotherapie fokussiert auf die der Symptomatik zugrunde liegenden Konflikte und strukturellen Defizite. Die spezifische therapeutische Haltung erlaubt es, speziell den interpersonellen Bereich spiegelnd, stützend und konfrontierend zu bearbeiten und beim Aufbau neuartiger Beziehungserfahrungen zu unterstützen. 


\section{Cave}

Fehler bei der Behandlung der akuten Depression bzw. am Beginn der analytischen und tiefenpsychologisch fundierten Psychotherapie:

- Es gilt, sowohl Überengagement als auch Distanzierung zu vermeiden.

- Durch die Hilflosigkeit, die Therapeuten häufig im Kontakt mit depressiven Patienten erleben, kann es leicht geschehen, dass sie, manchmal unausgesprochen, auf Besserung drängen, was aber einen erhöhten Gewissensdruck bei den Patienten zur Folge haben und damit beispielsweise zum Verschweigen von Symptomen führen kann.
- Die suggestive Aufforderung zu positiven Sichtweisen stellt eine Überforderung des Patienten dar.

- Spiegelung von Aggressivität kann dazu führen, dass sich der Patient noch hilfloser fühlt und auf diesem Weg Schuldgefühle und Autoaggression verstärkt werden.

- Besonders vorsichtig muss man bei der Suche nach auslösenden Ereignissen und problematischen biografischen Konstellationen vorgehen, da auch hier ein zu konfrontatives Vorgehen, ohne Anpassung an Befinden und Möglichkeiten des Patienten, zu erheblichen Schuldgefühlen und zum Verlust von als gut erlebten inneren Objekten führen kann.

\section{Stationäre psychodynamische Psychotherapie}

Schwere (v.a. suizidale) und chronische, insbesondere therapierefraktäre Depressionen sind oft nicht mehr ambulant zu behandeln und bedürfen einer stationären psychotherapeutischen Betreuung. Wir wollen die stationäre psychoanalytisch orientierte Therapie am Beispiel der Klinik für Psychosomatische Medizin und Psychotherapie am Städtischen Klinikum MünchenHarlaching darstellen, da beide Autoren mit dem dortigen Vorgehen vertraut sind und es gut vergleichbar ist mit anderen psychodynamisch orientierten Kliniken. Dort sind depressive Patienten mit etwa 40\% die deutlich größte Patientengruppe. Depression als komorbide Störung bei anderen Erkrankungen liegt bei etwa 80\% vor.

Kein spezifisches Setting. Wir behandeln depressive Patienten nicht in einem eigenen Setting, sondern auf allen Stationen und in unserer Tagesklinik. Selbstverständlich wird auf die für Depressive typischen Konflikte und Probleme (wie z. B. der Individuations-/Abhängigkeitskonflikt, Trennungs- und Aggressionsprobleme) in der Behandlung fokussiert, aber nicht im Rahmen einer Depressionsstation bzw. eines spezifischen Settings, so wie wir es für andere Störungsgruppen (z.B. Spezialsetting für Borderline-Persönlichkeitsstörung, für Essstörung, für Traumafolgestörung, vgl. [13]) tun.

Da bei einer stationären Aufnahme die Depression meistens besonders schwer und akut ist, bewähren sich hier zunächst die Behandlungsprinzipien, wie sie unter Behandlung der akuten Depression dargestellt wurden.
In der stationären Behandlung werden zunächst die Behandlungsprinzipien für die akute Depression angewandt.

Behandlungselemente. Formal handelt es sich um ein Behandlungssetting mit folgenden Behandlungselementen:

- 2-mal in der Woche psychodynamische Einzelgespräche

- 2-4 psychodynamische Gruppensitzungen pro Woche

- Milieutherapie in der Gruppe

- Bezugspflegegespräche

- allgemeinärztliche Betreuung

- Visiten

- Gestaltungstherapie in Gruppen- und Einzelsitzungen

- konzentrative Bewegungstherapie in Gruppen- und Einzelsitzungen

- Training der sozialen Kompetenz in der Gruppe

- Sport- und Kreativangebote

- verhaltenstherapeutische und übende Techniken wie Entspannungstherapie, Fertigkeitentraining und sozialpädagogische Beratung bei sozialen und beruflichen Problemen

- Paar-/Familiengespräche bei einem Großteil der Patienten

- Erstellung eines persönlichen Therapieplans für jeden Patienten

Gruppendynamik. Stationäre Psychotherapie wird sehr von Aspekten der Gruppendynamik mitbestimmt, da die Patienten einen großen Teil der Zeit mit Mitpatienten in einer Großgruppe verbringen. Der therapeutische Prozess auf der Station wird daher immer von der Gesamtgruppe, also den Mitpatienten und dem 
therapeutischen Team, mitgetragen. Daraus ergibt sich, dass neben der zentralen Bedeutung der Beziehung zum Einzeltherapeuten der Gruppenaspekt eine wichtige Rolle im therapeutischen Setting spielt.

Pflegeteam. Das Pflegepersonal ist ein sehr wichtiger Teil des therapeutischen Teams; der pflegerische Ansatz liegt im Symptommanagement, in der Förderung der kommunikativen Kompetenz, der Aktivierung von Ressourcen und in der Hilfestellung bei Schwierigkeiten in der Alltagsbewältigung. Jedem Patienten wird für die Dauer seines Aufenthaltes eine Bezugsperson aus dem Pflegeteam zugeteilt. In täglichen Teamsitzungen wird der fachliche Austausch zwischen den einzelnen Berufsgruppen gewährleistet.

Zeitlicher Rahmen. Die Aufenthaltsdauer liegt bei Patienten mit mittelschweren oder schweren Depressionen bei 8-12 Wochen. Damit sind 16-24 Einzelpsychotherapiesitzungen möglich, die mit einer mindestens gleichen Anzahl von Gruppentherapiesitzungen kombiniert werden.

Bei gut $80 \%$ der stationären Patienten ist es erforderlich, die Psychotherapie mit dem Ziel der Rezidivprophylaxe ambulant fortzuführen.

Im Sinne der Sicherung der Ergebnisqualität werden die Patienten zu Beginn und am Ende des stationären Aufenthaltes mit Selbsteinschätzungsfragebögen zu ihren psychischen Symptomen und ihren interpersonellen Problemen befragt. Die psychische Symptomatik verbessert sich dabei mit einer Effektstärke von 0,97, was in der Psychotherapieforschung als eine hohe Effektstärke gilt, also einem großen Effekt entspricht. Die Effektstärke für den Gesamtwert interpersoneller Probleme beträgt 0,55, das gilt als ein mittlerer Effekt [14].

\section{Stand der Psychotherapie- forschung zur Depressions- behandlung}

Für die psychodynamische Kurzzeitpsychotherapie zeigt die Metaanalyse von Driessen et al. für den Prä-/ Postvergleich eine hohe Effektstärke, für den Vergleich mit einer Warteliste-Kontrollgruppe oder „Care as usual“-Gruppe eine mittlere Effektstärke [15]; beides weist die psychodynamische Kurztherapie als eine wirksame Behandlungsform aus. Darüber hinaus gibt es empirische Hinweise, dass die Kurzpsychotherapien Rückfälle und Wiedererkrankungen nicht verhindern können. So zeigten Westen und Morrison in einer Metaanalyse von Kurzzeittherapien, dass nur etwa die Hälfte aller depressiven Patienten sich symptomatisch besserte und dass diese Besserung nur bei etwa einem Drittel in einem Zeitraum von 12-18 Monaten stabil blieb [16].

Vergleich Kurz-|Langzeittherapie. Das Problem der bleibenden Veränderung nach einer Psychotherapie, im Falle der depressiven Störung die Reduktion oder das Ausbleiben von weiteren depressiven Episoden, ist in das Zentrum der empirischen Psychotherapieforschung gerückt. Interessant in diesem Zusammenhang ist die Helsinki Psychotherapy Study, weil sie Ergebnisse von psychodynamischer und lösungsorientierter Kurzpsychotherapie mit denen einer psychodynamischen Langzeittherapie über mehrere Messzeitpunkte hinweg vergleicht [17]. Ein Jahr nach Behandlungsbeginn zeigten die beiden Kurzpsychotherapien deutlich geringere Werte in den Depressionsmaßen als die Langzeitpsychotherapie, 3 Jahre nach Behandlungsbeginn aber war die Langzeitpsychotherapie den Kurzzeitpsychotherapien statistisch signifikant überlegen, denn nur sie führte zu einer weiteren deutlichen Reduktion der depressiven Symptomatik.

Nach 3 Jahren zeigt sich in Studien eine Überlegenheit der Langzeitpsychotherapie gegenüber der Kurzzeittherapie.

Zu ähnlichen Ergebnissen kommt auch die Münchner Psychotherapiestudie, die die Effektivität von analytischer Psychotherapie, tiefenpsychologisch fundierter Psychotherapie und Verhaltenstherapie mit einem prospektiven, teilweise randomisierten, kontrollierten Prozess-Outcome-Design bei depressiven Patienten untersuchte [12,18]. Alle 3 Behandlungsformen waren sehr effektiv in der Behandlung der depressiven Störung. Im direkten Gruppenvergleich war die analytische Psychotherapie in der Besserung der sozialen Beziehungen, bei der strukturellen Veränderung und bei der Anzahl der noch vorhandenen Diagnosen zum 1-Jahres-Katamnesemesszeitpunkt der tiefenpsychologisch fundierten Psychotherapie und der Verhaltenstherapie statistisch signifikant überlegen; letztere zusätzlich noch in der Erreichung der individuellen Therapieziele. Im subjektiven Symptommaß fand sich ein Jahr nach Therapieende kein deutlicher Unterschied zwischen den 3 Therapieformen, zur 3-Jahres-Katamnese war aber die analytische Psychotherapie den anderen beiden auch in der Symptombesserung überlegen. 
Kernaussagen

- Die Depression ist eine prinzipiell rezidivierende, oft chronische Erkrankung.

- Psychodynamische Psychotherapie gilt als Oberbegriff für analytische Psychotherapie und tiefenpsychologisch fundierte Psychotherapie.

- Bei der analytischen Psychotherapie stehen die Analyse der aktuellen Beziehung zwischen Analytiker und Patient, das Wissen um die Übertragung, die Verwendung der Gegenübertragung und die deutende Tätigkeit (inklusive der Übertragungsdeutung) im Vordergrund.
- Hauptfokus der tiefenpsychologisch fundierten Psychotherapie ist der Aktualkonflikt; regressive Prozesse werden begrenzt, Bewältigungsstrategien unterstützt und es wird vorwiegend auf der interpersonellen Ebene gearbeitet.

- Kurztherapien, die lediglich auf eine Beseitigung der aktuellen Symptome abzielen, verlieren an Bedeutung.

- Die psychodynamischen Psychotherapien als Langzeitpsychotherapien haben sich auch bei schwierigem Krankheitsverlauf in der Depressionsbehandlung als sehr effektiv herausgestellt.
Ein direkter Vergleich 3 Jahre nach Therapieende zeigte die Überlegenheit der analytischen Psychotherapie gegenüber der tiefenpsychologischen Psychotherapie und der Verhaltenstherapie.

„Erhaltungspsychotherapie“. All diese Befunde und das durch epidemiologische Studien gut abgesicherte Wissen, dass die Depression nicht eine benigne Erkrankung ist, die auch ohne Behandlung einen günstigen Verlauf nähme, sondern eine meist rezidivierend oder chronisch verlaufende Erkrankung ist [19], haben zu der Schlussfolgerung geführt, eine langfristige, niederfrequente „Erhaltungspsychotherapie“, analog der Erhaltungsdosis bei der psychopharmakologischen Behandlung, einzuführen. Speziell einer Risikogruppe von depressiven Patienten, z.B. denen mit erheblicher Restsymptomatik, insbesondere mit Schlafstörungen, oder mit mehr als 3 vorausgegangenen depressiven Episoden, mit ausgeprägter Persönlichkeitsstörung u. a., sollte nach einer Kurzzeittherapie diese „Erhaltungspsychotherapie“ prinzipiell angeboten werden, statt erst dann zu reagieren, wenn es bereits zu einem Rückfall gekommen ist [20].

Aufgrund des chronischen Charakters der Depression bietet sich nach einer Kurzzeittherapie eine langfristige niederfrequente „Erhaltungspsychotherapie“ als Rezidivprophylaxe an.

Eine andere Konsequenz ist, depressive Patienten mit Langzeitpsychotherapien zu behandeln, die im Sinne eines „Carry-over“-Effektes Rückfälle verhindern können, indem sie die Patienten für das Erkennen typischer Anlasssituationen sensibilisieren und sie mit besseren Bewältigungsmechanismen ausstatten. Einen ausführlicheren Überblick zum Stand der Psychotherapieforschung in der Depressionsbehandlung geben Klug u. Huber [21].

\section{Über die Autoren}

\section{Dorothea Huber}

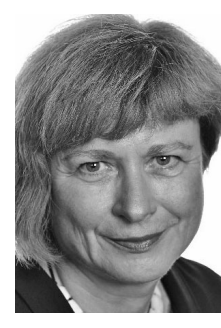

Prof. Dr. med. Dr. phil. Chefärztin an der Klinik für Psychosomatische Medizin und Psychotherapie, Klinikum München-Harlaching. Engagiert in Forschung und Lehre sowohl am Klinikum rechts der Isar, TUM, als auch an der International Psychoanalytic University Berlin. Sie hat die Münchner Psychotherapiestudie an Depressiven durchgeführt und ist an der DPG-Praxisstudie sowie der DGPTAngststudie beteiligt. Sie ist Mitglied der DPG-Forschungskommission und der DGPT-Forschungskonferenz.

\section{Günther Klug}

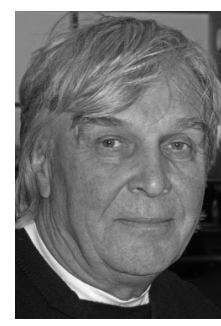

Dr. med. Er war Oberarzt an der Klinik für Psychosomatische Medizin und Psychotherapie, Klinikum München-Harlaching und ist Mitarbeiter am Klinikum rechts der Isar, TUM. Er hat die Münchner Psychotherapiestudie an Depressiven durchgeführt und ist Kontrollanalytiker für psychoanalytisch-modifizierte Verfahren an der Akademie für Psychoanalyse in München.

\section{Korrespondenzadresse}

Prof. Dr. med. Dr. phil. Dorothea Huber

Klinik für Psychosomatische Medizin und Psychotherapie Klinikum Harlaching

Sanatoriumsplatz 2

81545 München

E-Mail: dorothea.huber@klinikum-muenchen.de 


\section{Literatur}

1 DGPPN, BAEK, KBV et al. S3-Leitlinie/Nationale Versorgungsleitlinie Unipolare Depression-Langfassung. DGPPN, AEzQ, AWMF; 2009

2 Wittchen $\mathrm{H}-\mathrm{U}$, Müller N, Schmidtkunz B et al. Erscheinungsformen, Häufigkeit und Versorgung von Depressionen. Ergebnisse des bundesweiten Gesundheitssurveys „Psychische Störungen“. Fortschr Med 2000; (Suppl. 01): 4-9

3 Roth A, Fonagy P. What works for whom? New York, London: The Guilford Press; 2005

4 Rudolf G. Störungsmodelle und Interventionsstrategien in der psychodynamischen Depressionsbehandlung. Z Psychosomat Med Psychother 2003; 49: 377-390

5 Leuzinger-Bohleber M, Bahrke U, Beutel M et al. Psychoanalytische und kognitiv-verhaltenstherapeutische Langzeitpsychotherapien bei chronischer Depression: die LAC-Depressionsstudie. Psyche - Z Psychoanal 2010; 64: 782-832

6 Arbeitskreis OPD. Operationalisierte Psychodynamische Diagnostik OPD-2. Bern: Huber; 2006

7 Schauenburg H. Psychodynamische Psychotherapie. In: Schauenburg H, Hoffmann N, Hrsg. Psychotherapie der Depression. 2. Aufl. Stuttgart: Thieme; 2007: 45-64

8 Huber D, Will H. Psychoanalyse. In: Schauenburg H, Hoffmann N, Hrsg. Psychotherapie der Depression. 2. Aufl. Stuttgart: Thieme; 2007: 65-76

9 Strachey J. Die Grundlagen der Wirkung der Psychoanalyse. Int Z Psychoanalyse 1935; 21: 486-516

10 Hoglend P, Bogwald K-P, Amlo S et al. Transference interpretations in dynamic psychotherapy: do they really yield sustained effects? Am J Psychiatry 2008; 165: 763-771

11 Wallerstein RS. Forty-two lives in treatment. New York: The Guilford Press; 1986
12 Huber D, Henrich G, Clarkin J et al. Psychoanalytic versus psychodynamic therapy for depression - a three-year follow-up study. Psychiatry (submitted)

13 Huber D, von Rad M. Störungsorientierte psychodynamische Therapie im Krankenhaus. Stuttgart: Kohlhammer; 2011

14 Huber D, Albrecht C, Henrich G et al. Langzeit-Katamnese zur Effektivität einer stationären psychodynamischen Psychotherapie. Z Psychosomat Med Psychother 2009; 55: 189-199

15 Driessen E, Cuijpers P, de Maat SC et al. The efficacy of shortterm psychodynamic psychotherapy for depression: A metaanalysis. Clin Psychol Rev 2010; 30: 25-36

16 Westen D, Morrison K. A multidimensional meta-analysis of treatments for depression, panic, and generalized anxiety disorder: an empirical examination of the status of empirically supported therapies. J Consulting Clin Psychol 2001; 69: 875899

17 Knekt P, Lindfors O, Härkänen T et al. Randomized trial on the effectiveness of long- and short-term psychotherapy and solution-focused therapy on psychiatric symptoms during a 3year follow-up. Psychol Med 2008; 38: 689-703

18 Huber D, Henrich G, Gastner J et al. Must all have prices? The Munich Psychotherapy Study. In: Ablon JS, Levy R, Kaechele H, eds. Evidence Based Psychodynamic Psychotherapy. New York: Humana Press; 2011

19 Judd LL. The clinical course of unipolar major depressive disorders. Arch Gen Psychiatry 1997; 54: 989-991

20 Schauenburg H, Clarkin J. Rückfälle bei depressiven Erkrankungen - Sind psychotherapeutische „Erhaltungsstrategien“ sinnvoll? Z Psychosomat Med Psychother 2003; 49: 377-390

21 Klug G, Huber D. Psychotherapieforschung bei depressiven Störungen. In: Will H, Grabenstedt Y, Völkl G, Banck G, eds. Depression. Stuttgart: Kohlhammer; 2009: 39-46 


\section{CME.thieme.de}

\section{CME-Fragen}

\section{CME-Teilnahme}

- Viel Erfolg bei Ihrer CME-Teilnahme unter http://cme.thieme.de

- Diese Fortbildungseinheit ist 12 Monate online für eine CME-Teilnahme verfügbar.

- Sollten Sie Fragen zur Online-Teilnahme haben, unter http://cme.thieme.de/hilfe finden Sie eine ausführliche Anleitung.

\section{1}

Welche Aussage zum Verlauf der Depression ist richtig?
A Bei der Depression handelt es sich um eine meist wiederkehrende Erkrankung, die in 10-30\% der Fälle chronisch wird.

B Bei der Depression handelt es sich um eine Erkrankung mit meist einer Episode, die aber in $10-30 \%$ der Fälle chronisch wird.

C Bei der Depression handelt es sich um eine meist wiederkehrende Erkrankung, die kaum chronifiziert.

D In den meisten Fällen nimmt die Depression einen primär chronischen Verlauf.

E Bei der Depression handelt es sich um eine Erkrankung mit meist einer Episode, die nur selten chronisch wird.

\section{2}

Welche Aussage zur Nationalen Versorgungsleitlinie Depression ist richtig?
A Die Nationale Versorgungsleitlinie Depression ist auf der Grundlage einer konsensuellen Entscheidung deutscher Psychiater zustande gekommen.

B Die Nationale Versorgungsleitlinie Depression ist auf der Grundlage einer konsensuellen Entscheidung deutscher Psychotherapeuten zustande gekommen.

C Die Nationale Versorgungsleitlinie Depression gilt als Grundlage für die Kostenerstattung der psychodynamischen Therapie depressiver Patienten.

D Die Nationale Versorgungsleitlinie Depression wurde von ärztlichen und psychologischen Fachgesellschaften und Berufsverbänden erarbeitet.

E Die Nationale Versorgungsleitlinie Depression empfiehlt in erster Präferenz für alle Depressionsformen psychodynamische Psychotherapie.

3

Welche Aussage zur analytischen Psychotherapie ist richtig?
A Hauptfokus der Therapie ist der Aktualkonflikt.
B Hauptfokus der Therapie ist die Veränderung depressiver Denkmuster.
C Hauptfokus der Therapie ist die Arbeit an der Übertragung auf den Therapeuten.
D Die analytische Psychotherapie basiert auf der Verstärker-Verlust-Theorie.
E Alle Aussagen sind falsch.

A Beim akut Depressiven verbietet sich eine genaue Exploration der Symptomatik.

B Beim akut Depressiven soll das Suizidrisiko nicht angesprochen werden, um die Suizidalität nicht zu initiieren.

C Bei der Therapie der akuten Depression soll die Frage einer evtl. Begleitmedikation nicht angesprochen werden, um den Patienten nicht zu verunsichern.

D Klare Absprachen am Beginn einer Behandlung sind unbedingt erforderlich.

E Der Therapeut sollte nicht seine eigene Wahrnehmung zur Verfügung stellen, um Abhängigkeit zu vermeiden. 


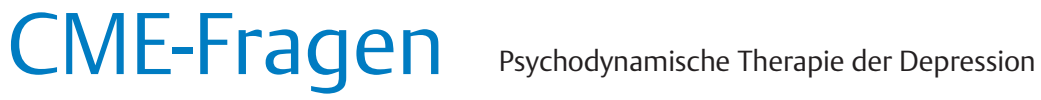

\section{6}

Welches der angegebenen Ziele der analytischen Psychotherapie ist falsch?
A die Analyse der negativen Übertragung

B aus der chronischen Enttäuschung die Enttäuschungswut befreien

C die destruktiven Schuldgefühle in eine zunehmende Fähigkeit zur aktiven Beziehungsgestaltung umwandeln

D die destruktiven Phantasien erlebbar machen und dadurch die in ihnen liegenden potenziell positiven Kräfte freilegen

E die Aggressivität des Patienten möglichst frühzeitig freilegen
Welche Aussage über die tiefenpsychologisch fundierte Psychotherapie bei Depressiven ist falsch?

A Das depressive Symptom steht im Vordergrund der Behandlung.

B Die gleichschwebende Aufmerksamkeit des Therapeuten wird durch eine aktive, fokussierte Haltung eingeschränkt.

C Die Aggressivität kann häufig nicht in der Übertragung auf den Therapeuten bearbeitet werden.

D Die Übertragungsdeutung als Intervention ist nicht zentraler Bestandteil der Technik.

E Die Arbeit an der Außenübertragung ist eine wichtige Behandlungsstrategie.

\section{8}

Welche Aussage zur stationären Psychotherapie der Depression ist richtig?
A Schwere und chronische Depressionen bedürfen häufig einer stationären psychotherapeutischen Betreuung.
B Depressive Patienten machen nur einen kleinen Teil in der Klientel der stationären Psychotherapie aus.
C Depressive Patienten werden überwiegend im einzeltherapeutischen Setting behandelt.
D Nach einer intensiven stationären Psychotherapie erübrigt sich eine ambulante Weiterbehand- lung.
E Die stationäre Psychotherapie Depressiver führt üblicherweise zu kleinen Effektstärken.

Welche Aussage zur Psychotherapieforschung ist für die Depression falsch?
A Die Kurzzeittherapie hat sich bei der Behandlung der Depression als ausreichend herausgestellt.

B Zur Einschätzung der Effektivität der Psychotherapie bei Depressiven ist eine längere Katamnese wichtig.

C Um die Effektivität der psychodynamischen Psychotherapie von Depressiven einschätzen zu können, muss mehr als nur die Symptomatik erfasst werden.

D Für die psychodynamische Psychotherapie von Depressiven werden signifikante Veränderungen dargestellt.

E Nach der Therapie weiterbestehende Symptomatik ist ein Indikator für Rückfälle.
Welche Aussage zur Ätiologie der Depression ist falsch?
A Der depressive Grundkonflikt ist durch Objektbedürftigkeit und Objektenttäuschung gekennzeichnet.

B Die Verarbeitung des depressiven Grundkonflikts ist durch die Bemühung, das Objekt zu erhalten, gekennzeichnet.

C Der Versuch sich das Objekt zu erhalten, führt zur Idealisierung.

D Der Versuch sich das Objekt zu erhalten, führt zur Wendung der Aggression gegen das eigene Selbst.

E Der depressive Grundkonflikt ist gekennzeichnet durch die Liebe zum gegengeschlechtlichen Elternteil und den Hass auf das gleichgeschlechtliche Elternteil. 